\title{
Antiallergic activities of shallot (Allium ascalonicum L.) and its therapeutic effects in allergic rhinitis
}

\author{
Warangkana Arpornchayanon, ${ }^{1}$ Soraya Klinprung, ${ }^{1}$ Sunee Chansakaow, ${ }^{2}$ Nutthiya Hanprasertpong, ${ }^{1}$ \\ Saisawat Chaiyasate, ${ }^{3}$ Masaaki Tokuda, ${ }^{4}$ Hirotoshi Tamura ${ }^{5}$
}

\section{Abstract}

Background: Onion has antiallergic activity but lack of evidence for shallot.

Objectives: To determine whether shallot owns similar antiallergic activity to onion and its therapeutic effects in allergic rhinitis when added to standard treatment

Methods: In-vitro $\beta$-hexosaminidase inhibitory activities of shallot was compared with onion on RBL-2H3 cells. In clinical study, a randomized, double-blind, placebo-controlled trial was performed. Sixteen AR patients were randomized equally into the controls who received cetirizine $10 \mathrm{mg}$ once daily and placebo capsules for 4 weeks, and the treatment who received $3 \mathrm{~g}$ of oral shallot per day (equivalent to $1 \frac{1 / 2}{2}$ bulbs) and cetirizine. Visual analog scores of overall symptoms (VAS), total nasal and ocular symptom scores (TNSS and TOSS), nasal airway resistance (NAR), and adverse events were assessed.

Results: Shallot extract at $200 \mu \mathrm{g} / \mathrm{mL}$ had an average $\beta$-hexosaminidase inhibition rate of 97\% while onion extract had 73\%. HPLC chromatograms $(\lambda=290 \mathrm{~nm})$ of both plants showed nearly identical patterns of quercetin compounds, such as quercetin 3,4'-diglucoside, quercetin $4^{\prime}$-glucoside, and quercetin. After 4-week of treatment, $62.5 \%$ of patients in shallot group and $37.5 \%$ of patients in control group showed improvement of post-treatment VAS. TNSS were significantly reduced in both groups, however no difference between groups $(P=0.18)$. TOSS were significantly improved only in the shallot group $(P=0.01)$. Adverse events from shallot were not different from placebo.

Conclusions: Shallot had antiallergic activity and similar quercetin compounds to onion. The shallot oral supplement and cetirizine was shown to improve the overall AR symptoms more than cetirizine alone.

Key words: Allergic rhinitis, onion, shallot, Allium ascalonicum L., Allium cepa

\section{Citation:}

Arpornchayanon, W., Klinprung, S., Chansakaow, S.,

Hanprasertpong, N., Chaiyasate, S., Tokuda, M., Tamura, H.

(2022). Antiallergic activities of shallot (Allium ascalonicum L.)

and its therapeutic effects in allergic rhinitis.

Asian Pac J Allergy Immunol, 40(4), 393-400.

https://doi.org/10.12932/ap-300319-0529

\footnotetext{
Affiliations:

${ }^{1}$ Department of Pharmacology, Faculty of Medicine,

Chiang Mai University, Thailand

${ }^{2}$ Department of Pharmaceutical Sciences and Medicinal Plant Innovation Center, Faculty of Pharmacy, Chiang Mai University, Thailand

${ }^{3}$ Department of Otolaryngology, Faculty of Medicine, Chiang Mai University, Thailand

${ }^{4}$ Faculty of Medicine, Kagawa University, Japan

${ }^{5}$ Department of Applied Biological Science, Faculty of Agriculture, Kagawa University, Japan
}

\section{Corresponding author:}

Warangkana Arpornchayanon

Department of Pharmacology, Faculty of Medicine,

Chiang Mai University, Chiang Mai 50200, Thailand

E-mail: warangkana@gmail.com

\section{Introduction}

Allergic rhinitis (AR) is a global health problem that affects over 400 million people worldwide. ${ }^{1}$ Pathophysiology of AR involves IgE-mediated immunological response against specific inhaled allergens, leading to the releases of chemical mediators such as leukotrienes, prostaglandins, and histamine. ${ }^{2}$ These mediators stimulate mast cells and eosinophils function which induce inflammation of nasal mucosa. Following initial sensitization, symptoms that occur during the early phase of re-exposure to aeroallergens include sneezing, itchy nose, nasal congestion, and rhinorrhea. 
The late-phase symptoms, for example, nasal obstruction, are usually a result of cellular infiltration or chronic inflammation of nasal mucosa. ${ }^{3}$ Various medicinal plants have been used in Complementary and Alternative Medicine (CAM) to reduce nasal symptoms of AR. ${ }^{4-7}$ For example, red shallots or Allium ascalonicum $L$. are recommended in Thai traditional medicine to prevent itchy and runny nose while onions or Allium cepa are used in Japanese remedy for the relief of nasal congestion. ${ }^{8}$

Shallots and onions are in the same Alliaceae family as garlics. ${ }^{9-10}$ Both of them are perennial crops that grow annually in clusters of small bulbs and cloves. However, their external appearances, scent, and taste differ. A.ascalonicum L. looks similar to A.cepa in form of bulb-multiplying layers but smaller in size and mostly found in Southeast Asia. Shallot delivers stronger odor than onion because of the higher amount of sulfur compounds. It has delicate onion-like flavor but sweeter. It has been used to treat many diseases since ancient history in Thailand and Indian Ayurvedic Medicine. ${ }^{11}$ Shallot is best-known as an important ingredient of the famous Thai cuisine, 'Tom Yam Koong. There are reports of anti-oxidant, anti-inflammatory, and anti-fungal properties of shallots in the in vitro and in vivo studies. ${ }^{11-15}$ Shallot can also reduce serum level of total cholesterol and low-density lipoprotein (LDL). ${ }^{13,15}$

Shallot contains high levels of fats and soluble solids, including sugars together with sulfur-containing compounds, such as S-allyl cysteine sulfoxide that gives strong odor. ${ }^{9}$ Dried shallot comprises $70-85 \%$ of carbohydrates which are mainly glucose, fructose, and sucrose. In $100 \mathrm{~g}$ of its fresh weight, the components comprise $79.8 \mathrm{~g}$ of, $72 \mathrm{~g}$ of calories, $2.5 \mathrm{~g}$ of protein, $0.1 \mathrm{~g}$ of fat, $16.8 \mathrm{~g}$ of carbohydrate, and $0.7 \mathrm{~g}$ of fiber. The vitamins and minerals component consist of 37 $\mathrm{mg}$ of calcium, $60 \mathrm{mg}$ of phosphorous, $1.2 \mathrm{mg}$ of Iron, $12 \mathrm{mg}$ of sodium, $334 \mathrm{mg}$ of potassium, $0.06 \mathrm{mg}$ of thiamine, 0.02 $\mathrm{mg}$ of riboflavin, $0.2 \mathrm{mg}$ of niacin, and $8 \mathrm{mg}$ of vitamin C. ${ }^{16-17}$ Total phenolic content (TPC) in fresh shallot is approximately $2,528 \pm 43 \mathrm{mg}$, which seems to be the highest among plants in the Alliaceae family. ${ }^{10}$ High redox potential of shallot correlates with its robust anti-oxidant property. ${ }^{17-18}$

Correspondingly to onion, chemical analysis of shallot extract demonstrates the presence of flavone and polyphenolic derivatives such as quercetin, quercetin $4^{\prime}$-glucoside, quercetin 7,4'-diglucoside, quercetin 3,4'-diglucoside, quercetin aglycone, quercetin mono-D-glucose, isorhamnetin 3,4'-diglucoside, and isorhamnetin $4^{\prime}$-glucoside. ${ }^{19-23}$ However, no definite comparison has been made to the quercetin amounts between these two plants. The possible mechanism of action of shallot in allergic control could be the quercetin action, which stabilizing the mast cells from degranulation during the allergic response. ${ }^{24}$

There are many kinds of vegetables and fruits that have been tested and positive for antiallergic activities, for instance, sweet potatoes, lotus, mints, and onions. Onion is one of the plants in Liliaceae family along with shallot that the anti-allergic activity has been shown. Recently, the antiallergic activities of quercetin and quercetin-4'-glucoside in onion have been confirmed via QuEChERS (Quick, Easy, Cheap, Effective, Rugged, and Safe) method, but there is no data available for that of shallot. ${ }^{23}$ Hence, the primary purpose of this study was to investigate the antiallergic activity of shallot by using the similar assay described by Sato et al. ${ }^{23}$ and compared the $\beta$-hexosaminidase inhibition rates of type I allergy on Rat Basophilic Leukemia cell line (RBL-2H3 cells) between shallot and onion. After the antiallergic activity of shallot had been confirmed in the in-vitro phase, the preliminary clinical trial in AR patients was carried out. The aims of this study were to determine whether shallot owns antiallergic property similarly to onion and benefits the allergic treatment when given in combination with cetirizine for AR patients.

\section{Methods \\ In-vitro analysis}

For in-vitro study, chemical and biological analyses were performed at the Department of Applied Biological Science, Faculty of Agriculture, Kagawa University, Japan. Cultivars of shallot were collected from Chiang Mai (Northern, Thai), Thailand, while the onions (Satsuki, Japanese) were purchased in Kagawa, Japan. The analytical assay was followed steps by steps as described by Sato et al. ${ }^{23}$

Both shallots and onions were cleansed and the outer layers were peeled. Ten grams of each plants were weighted, then blended by a food processor into small pieces. After that, the extraction process began by using the QuEChERS method, ${ }^{23}$ Aceonitrile $10 \mathrm{~mL}$ was added into the specimen and homogenize was allowed via $10000 \mathrm{rpm}$ of centrifuged. Sodium chloride $1 \mathrm{~g}$, trisodium citrate dehydrate $1 \mathrm{~g}$, disodium hydrogen citrate sesquihydrate $0.5 \mathrm{~g}$, anhydrous magnesium sulfate $4 \mathrm{~g}$ were added, then hand-shaking for 1 minute. The specimen was then centrifuged at $3000 \mathrm{rpm}$ for 5 minutes. The acetonitrile extract was obtained and dried out by the evaporator. The next step was the extraction for antiallergic flavonoids from shallot and onion. The $200 \mu \mathrm{g} /$ $\mathrm{mL}$ concentration of shallot and onion extract were prepared for the antiallergic activity testing. Methanol was added into dry extract then diluted. The solvent was then removed by evaporator. Four $\mu \mathrm{L}$ of $100 \%$ DMSO and $3,966 \mu \mathrm{L}$ of MT buffer were added into each vial. Meanwhile, the Rat Basophilic Leukemia cell lines (RBL-2H3 cells) were prepared. Determination of Antiallergic activity was done by measuring the amount of $\beta$-hexosaminidase released by RBL-2H3 cells, using the ratio of $\beta$-hexosaminidase release (\%) after the IgE antibody had been prepared. The ratio of $\beta$-hexosaminidase of control should be greater than $25 \%$. The inhibition rate was calculated from the percentage of $\beta$-hexosaminidase release using the following formulas.

$$
\begin{aligned}
& \begin{array}{c}
\text { Ratio of } \\
\begin{array}{c}
\text {-hexosaminidase } \\
\text { release }(\%)
\end{array}
\end{array}=\frac{\left(\mathrm{OD}_{\text {control }} \text { or } \mathrm{OD}_{\text {sample }}-\mathrm{OD}_{\text {blank }}\right)}{\left(\mathrm{OD}_{\text {total }}-\mathrm{OD}_{\text {blank }}\right)} \times 100 \\
& \beta \text {-hexosaminidase release }(\%)=\frac{\mathrm{OD}_{\text {sample }}-\mathrm{OD}_{\text {blank }}}{\mathrm{OD}_{\text {control }}-\mathrm{OD}_{\text {blank }}} \times 100 \\
& \beta \text {-hexosaminidase inhibitory }(\%)=1-\frac{\mathrm{OD}_{\text {sample }}}{\mathrm{OD}_{\text {control }}} \times 100
\end{aligned}
$$


Next, HPLC analysis was performed with similar methods described by Tamura et al. ${ }^{23,25}$ The Chromatograms were obtained from UV absorbance at $290 \mathrm{~nm}$. The JASCO HPLC system (Tokyo, Japan) comprised a $250 \times 4.6 \mathrm{~mm}$ COSMOSIL 5C18-ARII column (Nacalai Tesque Inc., Kyoto, Japan) coupled to a JASCO MD 2010 Plus photodiode array detector and double JASCO PU-980 pumps with column temperature at $40^{\circ} \mathrm{C}$.

\section{Clinical Trial Study Design}

The prospective, randomized, double-blinded, parallel, controlled-trial, preliminary study was performed at Department of Pharmacology, Faculty of Medicine, Chiang Mai University, Thailand. The trial registry number was ChiCTR-IIR-17013331. The AR patients who met the inclusion criteria were enrolled and divided into 2 groups using the computerized block randomization. Each patient received generic cetirizine dihydrochloride tablets (ZERTINE $^{\oplus}$, Farmaline Ltd., Thailand) $10 \mathrm{mg}$ once daily for 4 weeks. Adjunctive treatment was applied to the patients in shallot group including the oral shallot supplement capsules of $3 \mathrm{~g}$ (equivalent to $1 \frac{1}{2}$ bulb) daily for 4 weeks. The patients in placebo group received similar amounts of identical capsules (starch with shallot odor) $3 \mathrm{~g}$ per day. Shallot and placebo capsules were prepared by the pharmacists in the Department of Pharmaceutical Science, Faculty of Pharmacy, Chiang Mai University, Thailand, which followed the quality standard control of Thai FDA guidelines for herbal products. Before the study began, the protocol was approved by the Human Research Ethics Committee of the Faculty of Medicine, Chiang Mai University, Thailand (IRB No. 254/2017). During the study, assessment of compliance was done by counting the left-over medication at every visit (= 2 weeks interval).

\section{Participants}

All patients gave their written informed consent prior to participation in the study. The inclusion criteria were male or female volunteers, aged 18 to 65 years old with positive skin prick test of at-least 1 allergen based on the common allergens in Thailand. The following 10 solutions were tested in each patient: histamine solution (positive control), glycerinate phenol-saline (negative control), house mite D. farina, house mite D. pteronyssinus, American cockroach, careless weed, para grass, Cladosporium spp., dog hair, and cat hair. Patients who had total nasal symptom score (TNSS) $\geq 6$ within prior 2 weeks with nasal congestion score $\leq 2$ were included into the study. After enrolment, they were firmly instructed to avoid foods or any supplements that contained shallots, onions, and garlics throughout the entire study period in order to avoid additional toxicities and confounding factors. Verbal assessment was used for reassurance in the second and third visits. Routine saline nasal irrigation was firmly instructed but topical/oral decongestants were not allowed.
The exclusion criteria were patients who had severe nasal anatomical abnormality (for example, nasal septum deviation $>50 \%$, nasal polyposis, and tumor), recent infection of the respiratory tract within 2 weeks, oral/intranasal corticosteroids or nasal surgery within 4 weeks before the study, immunotherapy, immunosuppressant, history of hypersensitivity to cetirizine or shallot, heart diseases, pregnancy, breastfeeding, hepatic and renal diseases. Patients whose serum aspartate aminotransferase (AST), alanine aminotransferase (ALT) elevation $>1.5$ times of the upper limit of normal range or estimated glomerular filtration rate (eGFR) $<50 \mathrm{ml} / \mathrm{min}$ were also excluded.

\section{Outcome measurements}

For subjective evaluation, a $100-\mathrm{mm}$ of visual analog scale of overall symptoms scores (VAS) was used to assess the patients' overall symptoms as a response to treatment in both groups. The score was rated by the patients before (pre-treatment) and at the end of the treatment (post-treatment). Scoring of $0(=0 \mathrm{~mm})$ meant subject had absolutely no AR symptom and score 10 (= $100 \mathrm{~mm})$ referred to the most severe AR symptoms. Favorable improvement of VAS referred to the more-than-50\% improvement of post-treatment VAS compared with pre-treatment. The total nasal symptom score (TNSS) included rhinorrhea, sneezing, itchy nose, and nasal congestion. Fully controlled of AR would be considered if the post-treatment TNSS $=0$ to 2 . Total ocular symptom score (TOSS) included watery eyes, itchy eyes, and eye redness. The evaluation criteria were as follows: $0=$ Absent (No symptom), 1 = Mild (Symptom was presented but not troublesome.), 2 = Moderate (Symptom was troublesome but did not interfere with normal activity.), 3 = Severe (Symptom disturbed normal activity). For objective evaluation, nasal airway resistance (NAR) was used to measure the nasal obstruction. Data from anterior rhinomanometry (PC 300, ATMOS, Germany) with the trans-nasal pressure at 75 Pascal (Pa) were collected at pre-treatment and post-treatment. Any adverse event that occurred during the treatment period were recorded.

\section{Statistical analysis}

For statistical analysis, values were represented in means \pm SD. Significant differences were considered when $P$-value $<0.05$. The test was used to confirm whether the antiallergic compounds in shallot is quercetin and the antiallergic activity in crude extracts of shallot was non-inferiority to onion. For within group comparison, mean change in VAS, TNSS, TOSS, and NAR from pre-treatment were analyzed by paired t-test. For between groups, Student's t-test was used to determine the differences of VAS, TNSS, TOSS and NAR. Percentages of responders were compared between groups using Chi's square. $P$-value of less than 0.05 was considered significant. 


\section{Results}

Antiallergic Activities of Shallot and Onion

Both shallot (Northern, Thai) and onion (Satsuki, Japanese) extracts had $\beta$-hexosaminidase release below $20 \%$ at $200 \mu \mathrm{g} / \mathrm{mL}$ of sample concentration. Shallot extract showed higher antiallergic activity than the onion (Table 1). An average relative inhibition rate of the shallot extract was $97 \%$ while the enzyme inhibition rate of the onion extract was $73 \%$. The HPLC chromatograms $(\lambda=290 \mathrm{~nm})$ of both shallot and onion demonstrated nearly identical patterns of quercetin compounds, such as quercetin 3,4'-diglucoside, quercetin $4^{\prime}$-glucoside, and quercetin (Figure 1).
Table 1. Antiallergic Activities of Shallot Versus Onion Samples.

\begin{tabular}{|l|c|c|}
\multirow{2}{*}{$\begin{array}{c}\text { QuEChERS Extract of Crude } \\
\text { Samples at } 200 \mu \mathrm{g} / \mathrm{mL}\end{array}$} & $\begin{array}{c}|c| \\
\text { Anti-allergic activity }\end{array}$ \\
\cline { 2 - 3 } $\begin{array}{c}\boldsymbol{\beta} \text {-hexosaminidase } \\
\text { release (\%) }\end{array}$ & $\begin{array}{c}\text { Relative Enzyme } \\
\text { inhibition (\%) }\end{array}$ \\
\hline Shallot (Northern, Thai) & $2.8 \pm 0.5$ & $97.15 \pm 1.3$ \\
Onion (Satsuki, Japanese) & $18 \pm 0.9$ & $73.98 \pm 2.0$ \\
\hline
\end{tabular}

Each value represents Mean \pm S.D. $(n=4)$
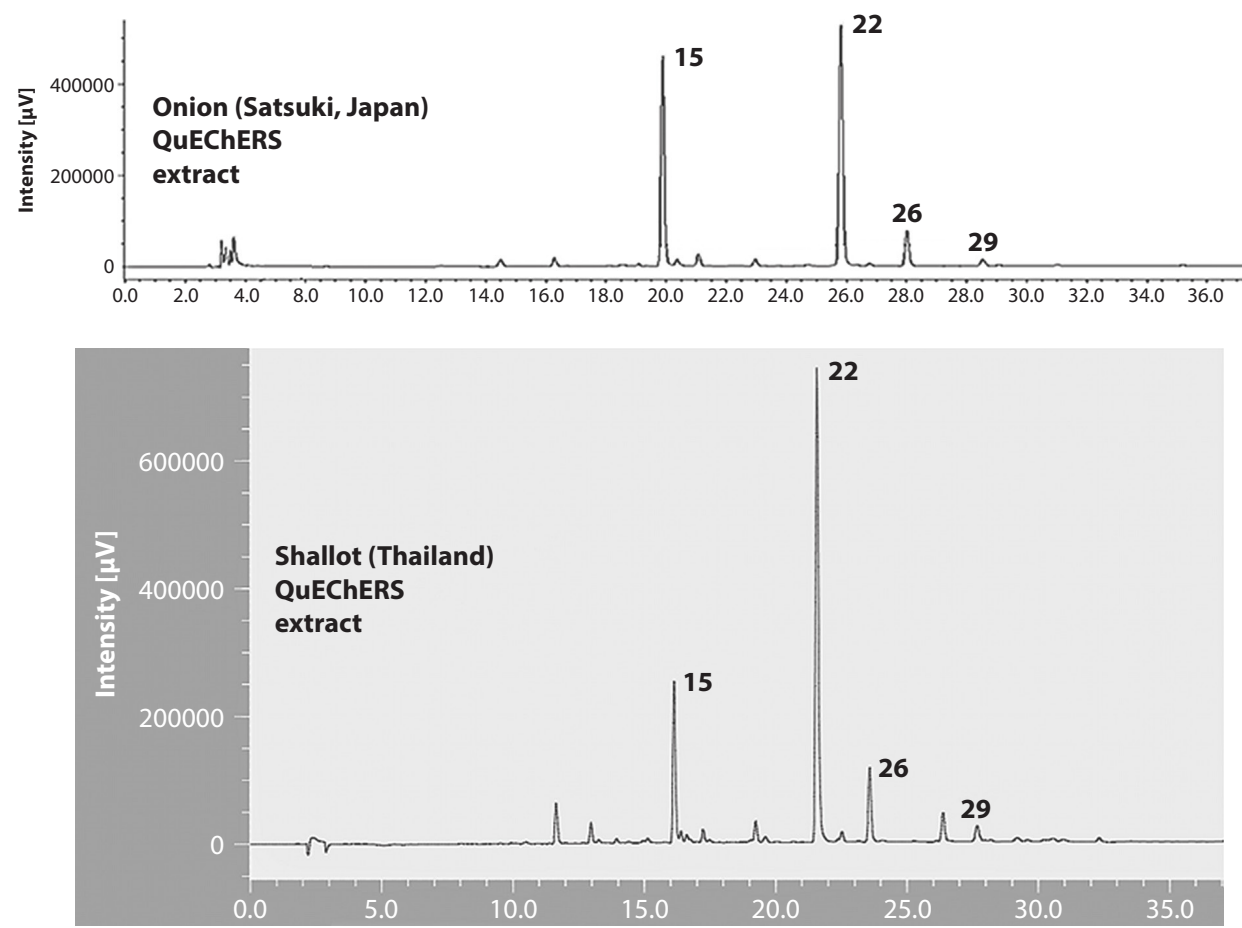

Identification of Compounds

- Compound $15=$ quercetin 3,4'-diglucoside

- Compound 22 = quercetin $4^{\prime}$-glucoside

- Compound 26 = isorhamnetin 4'-glucoside

- Compound 29 = quercetin

Figure 1. Similar HPLC Chromatograms of QuEChERS extracts of Japanese Onion (above) and Thai Shallot (below) at $\lambda=$ 290 nm. Retention Time and UV spectra of Each Peak with Authentic Compounds Were Identified.

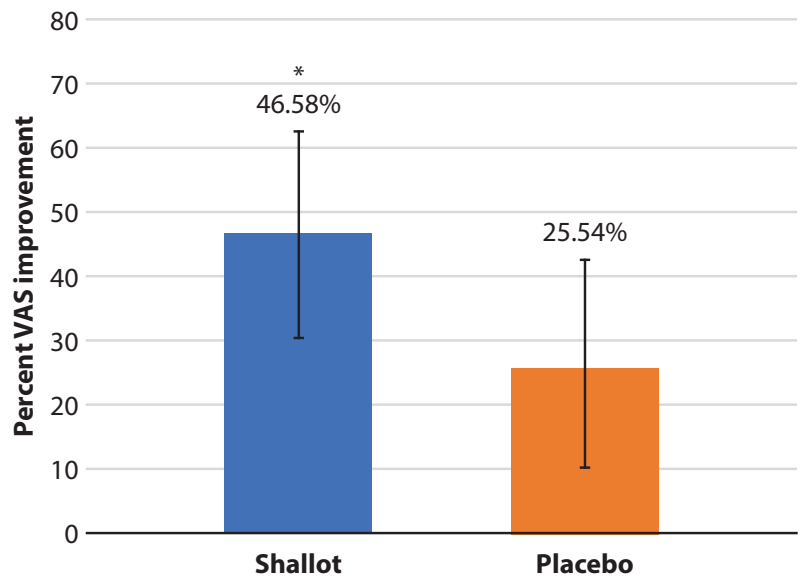

Figure 2. Percentage of VAS improvement at Post-treatment of Shallot and Placebo Groups.

* Significant Difference from Pre-treatment, $P<0.05$

\section{Therapeutic Effects of Shallot in AR}

The preliminary results of sixteen AR patients were presented in this study. All patients were randomized into shallot group $(n=8)$ and placebo group $(n=8)$. The most common allergens from skin prick test were house dust mites, D. farina and D. pteronyssinus, followed by American cockroaches. There were 7 patients in the shallot group and 8 patients in the placebo group who had associated ocular symptoms. Despite the age difference between groups, the mean duration of AR symptoms did not differ significantly.

After 4 weeks of treatment, 5 patients $(62.5 \%)$ in the shallot group and 3 patients $(37.5 \%)$ in the placebo group showed favorable improvement of VAS. The average of VAS at pre-treatment were $5.06 \pm 1.52$ in the shallot group and $5.38 \pm 2.76$ in the placebo group. Average VAS at post-treatment were $2.44 \pm 1.57$ in the shallot group and $3.98 \pm 2.81$ in the placebo group (Table 2). Average VAS was significantly decreased by $-46.58 \pm 34.66 \%$ only in the shallot group $(P=0.018)$ compared with $-25.54 \pm 34.30 \%$ in the placebo group $(P=0.193)$. 
Table 2. Mean VAS at Pre- and Post-treatment.

\begin{tabular}{|l|c|c|c|}
\hline Group & $\begin{array}{c}\text { Pre-treatment } \\
(\text { mean } \pm \text { SD) }\end{array}$ & $\begin{array}{c}\text { Post-treatment } \\
\text { (mean } \pm \text { SD) }\end{array}$ & P-value \\
\hline Shallot & $5.06 \pm 1.52$ & $2.44 \pm 1.57^{\star}$ & 0.018 \\
\hline Placebo & $5.38 \pm 2.76$ & $3.98 \pm 2.81$ & 0.193 \\
\hline
\end{tabular}

* Significant Difference from Pre-treatment, $P<0.05$

For nasal symptoms, patients in both groups showed significant improvement of TNSS after treatment, shown in 'Figure 3a'. In the shallot group, average TNSS was reduced from $8.00 \pm 1.85$ at baseline to $3.00 \pm 2.27$ at post-treatment $(P=0.002)$. Likewise, average TNSS was reduced from 7.00 \pm 1.07 pre-treatment to $4.00 \pm 2.73$ post-treatment in the placebo group $(P=0.021)$. However, there was no statistical difference between groups $(P=0.18)$.

For the associated ocular symptoms, only the patients who received oral shallot supplement in combination with cetirizine showed significant improvement of TOSS after treatment (Figure $3 \mathbf{b}$ ). In the shallot group, the average TOSS was reduced from $3.38 \pm 2.56$ at baseline to $0.38 \pm 0.52$ at post-treatment $(P=0.01)$. Meanwhile, average TOSS in the placebo group was reduced from $2.13 \pm 1.73$ pre-treatment to $1.13 \pm 1.73$ post-treatment $(P>0.05)$.

(3a)

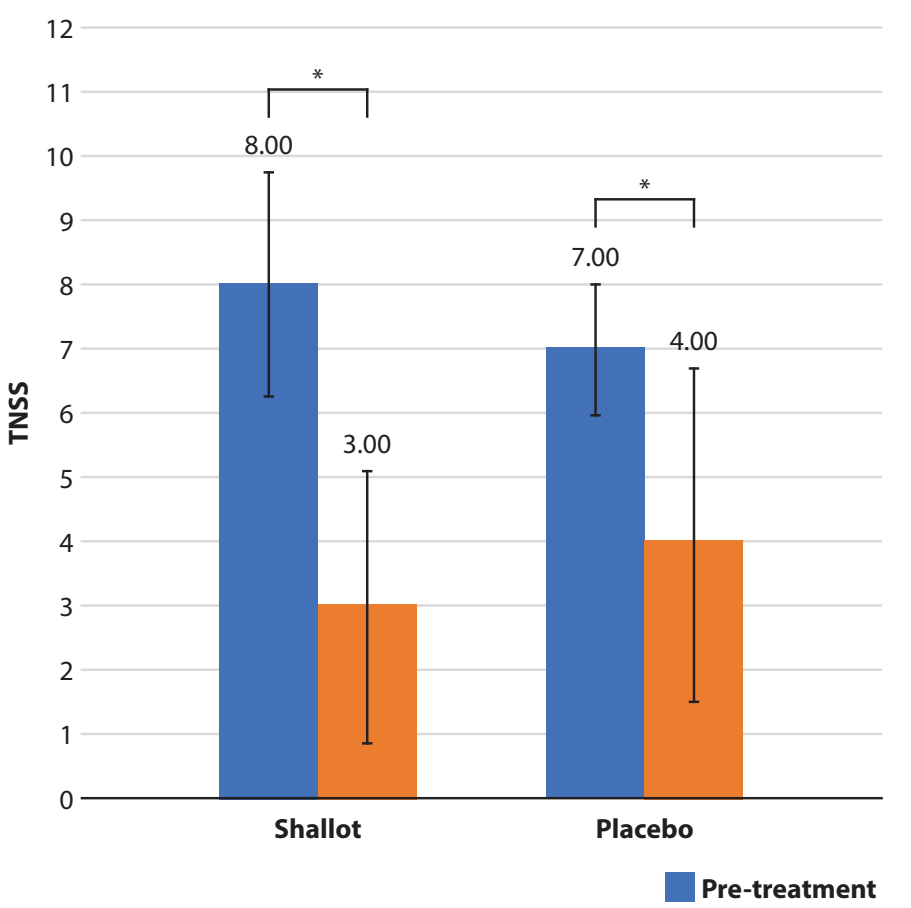

Sub analyses of nasal and ocular symptoms were shown in 'Table 3'. Sneezing, rhinorrhea, itchy nose and eyes were the main symptoms that markedly decreased after the treatment for 4 weeks. Scores of itchy nose and eyes were reduced significantly only in the shallot group $(P=0.007$ and $P=$ 0.003). Meanwhile, the post treatment scores of sneezing and rhinorrhea were improved in both groups but without statistically significant between groups. There was no change of other allergic symptoms such as nasal obstruction, watery eyes, and eye redness after treatment. There was no change of NAR after treatment in both groups. Average post treatment NAR were $0.27 \pm 0.16$ in the shallot group and $0.32 \pm 0.30$ in the placebo group.

All adverse events (AE) that occurred during treatment comprised dizziness, fatigue, headache, somnolence, rashes, decreased strength of hair root, nausea and dyspepsia. No serious AE was reported. There was no difference of AEs between the shallot and placebo groups. Adverse gastrointestinal effects occurred in the first to second weeks and gradually disappeared at the end of the study in both groups. The CNS adverse effects occurred immediately within the first week, remained, and disappeared approximately 1 week after the patients stop using all medication (on verbal follow-up). The skin adverse effects varied. The symptoms appeared within 2 weeks of treatment. The patients had their rashes disappeared on the third week of study while the cases with decreased strength of hair roots had their symptoms disappeared between 1 to 3 weeks of stop using medication.

(3b)

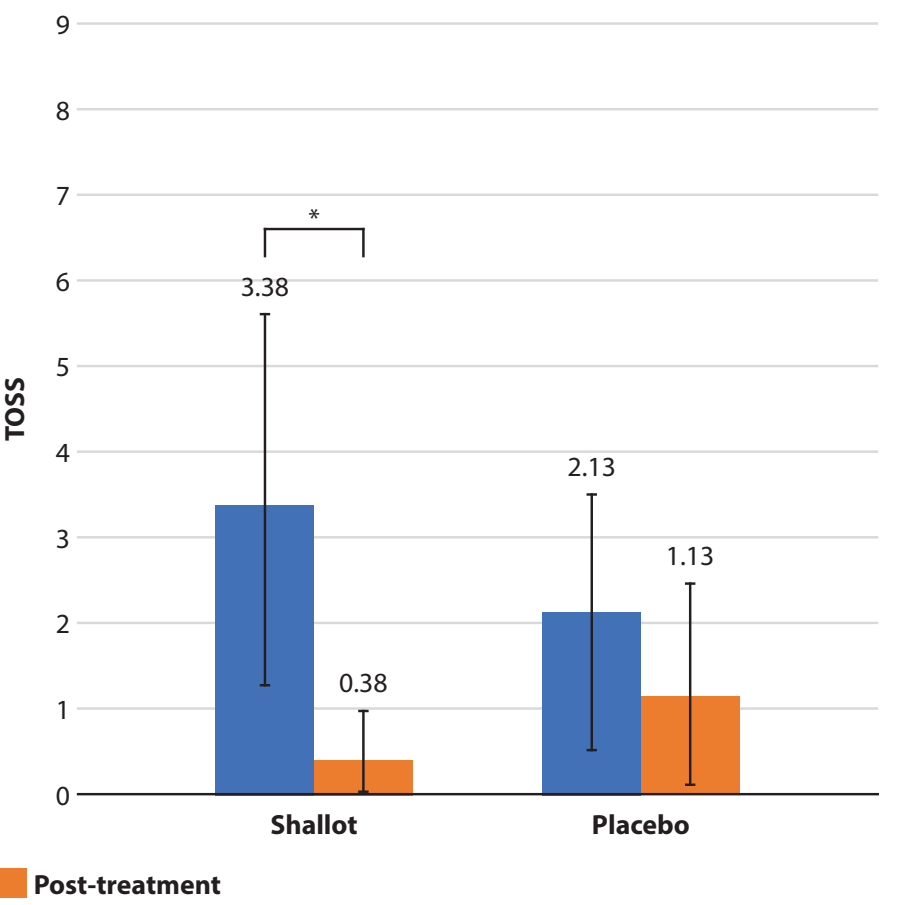

Figure 3. Mean TNSS at Pre- and Post-treatment (3a) and Mean TOSS (3b) of Shallot and Placebo Groups.

* Significant Difference from Pre-treatment, $P<0.05$ 
Table 3. Summary of Nasal and Ocular Symptoms Scores at Pre- and Post-treatment in Both Groups.

\begin{tabular}{|c|c|c|c|c|}
\hline Symptoms & $\begin{array}{l}\text { Pre-treatment } \\
(\text { mean } \pm \text { SD })\end{array}$ & $\begin{array}{l}\text { Post-treatment } \\
(\text { mean } \pm \text { SD })\end{array}$ & $\begin{array}{c}\% \text { change from } \\
\text { Pre-treatment } \\
(\text { mean } \pm \text { SD })\end{array}$ & $P$-value \\
\hline \multicolumn{5}{|l|}{ Nasal symptoms } \\
\hline \multicolumn{5}{|l|}{ Sneezing } \\
\hline Shallot & $2.13 \pm 0.64$ & $0.63 \pm 0.74^{*}$ & $-75.00 \pm 28.17^{*}$ & $<0.001$ \\
\hline Placebo & $1.38 \pm 0.52$ & $0.50 \pm 0.76^{*}$ & $-56.25 \pm 72.89^{\star}$ & 0.041 \\
\hline \multicolumn{5}{|l|}{ Rhinorrhea } \\
\hline Shallot & $2.38 \pm 0.74$ & $1.00 \pm 0.93^{*}$ & $-60.42 \pm 37.73^{\star}$ & 0.004 \\
\hline Placebo & $2.25 \pm 0.46$ & $1.00 \pm 0.53^{*}$ & $-54.17 \pm 27.82^{\star}$ & 0.002 \\
\hline \multicolumn{5}{|l|}{ Itchy nose } \\
\hline Shallot & $1.63 \pm 0.74$ & $0.50 \pm 0.53^{*}$ & $-64.58 \pm 44.04^{*}$ & 0.007 \\
\hline Placebo & $1.63 \pm 0.52$ & $1.00 \pm 0.93$ & $-37.50 \pm 51.75$ & 0.095 \\
\hline \multicolumn{5}{|l|}{ Nasal obstruction } \\
\hline Shallot & $1.75 \pm 0.46$ & $1.00 \pm 0.93$ & $-37.50 \pm 69.44$ & 0.080 \\
\hline Placebo & $1.88 \pm 0.35$ & $1.50 \pm 1.20$ & $-25.00 \pm 59.76$ & 0.351 \\
\hline \multicolumn{5}{|c|}{ Ocular symptoms } \\
\hline \multicolumn{5}{|l|}{ Watery eyes } \\
\hline Shallot & $0.75 \pm 0.89$ & $0.38 \pm 0.52$ & $-58.33 \pm 49.20$ & 0.351 \\
\hline Placebo & $1.00 \pm 1.31$ & $0.50 \pm 0.76$ & $-46.67 \pm 50.60$ & 0.275 \\
\hline \multicolumn{5}{|l|}{ Itchy eyes } \\
\hline Shallot & $1.50 \pm 0.93$ & $0.00 \pm 0.00^{*}$ & $-100.00 \pm 0.00^{*}$ & 0.003 \\
\hline Placebo & $1.25 \pm 0.46$ & $0.50 \pm 0.93$ & $-62.50 \pm 74.40$ & 0.048 \\
\hline \multicolumn{5}{|l|}{ Eye redness } \\
\hline Shallot & $0.50 \pm 0.76$ & $0.00 \pm 0.00$ & $-100.00 \pm 0.00$ & 0.104 \\
\hline Placebo & $0.50 \pm 0.76$ & $0.13 \pm 0.35$ & $-66.67 \pm 57.70$ & 0.197 \\
\hline
\end{tabular}

* Significant Difference from Pre-treatment, $P<0.05$

\section{Discussion}

Long-term use of antiallergic medication is necessary for allergic patients. ${ }^{26}$ There are many therapeutic options available including, allergen avoidance, pharmacotherapy, immunotherapy, surgery, and CAM. Limitations due to drugs safety and financial cost, non-pharmocological therapy such as CAM has become more popular. CAM such as acupuncture, aromatherapy, yoga, homeopathy, nasal irrigation, steam inhalation, and herbal supplement are commonly used. ${ }^{6}$ There are reports that herbs such as Petasites hybridus (butterbur), Chinese herbal medicine, Urticadioca (stinging nettle), Sambucus nigra (elderberry) and Nigella sativa (black seed) can reduce symptoms of AR. ${ }^{4,27}$ Some herbs are well known for their antiallergic activities, for example, onions and shallots. ${ }^{23}$ Shallot is one of Thai herbs that have been recommended for prevention of AR symptoms. ${ }^{8}$ Results from the in vitro part of this study has confirmed that the shallot had superior antiallergic activity than that of the onion despite similar composition.
However, different shallot/onion cultivars may have biological variations.

Similar to other plants in the Liliaceae family, there are many components in shallot, for example, allylsulfides, polyphenol, flavonoids especially quercetin, and sulfur compounds. ${ }^{10,28}$ Quercetin (3,3',4',5,7-pentahydroxyflavone) might be one of the key factors in both shallot and onion compounds. Quercetin is a polyphenol which belongs to the 'flavonoids', called 'flavonol'. Quercetin is found in a variety of foods including onions, shallots, apples, grapes, berries, broccoli, cherries, citrus fruits, and tea. However, the best food sources of quercetin seem to be the onions and shallots. ${ }^{29,30}$

There are many pharmacological effects of quercetin, including anti-allergic activity through antioxidant activities, inhibition of histamine and other chemical mediators, as well as stabilization of the mast cells. ${ }^{20-21,24}$ The antiallergic and anti-inflammatory properties of quercetin help preventing 
the symptoms of $\mathrm{AR}$ and asthma in allergic patients. The mechanism of action is possibly via the inhibition of histamine release and production, with modulating intracellular calcium levels and phosphokinase-C activation. ${ }^{31}$ Moreover, quercetin can also provide antiallergic activity by stimulation of immune system, decreasing proinflammatory cytokines, suppressing interleukin (IL-4), and improving the Th1/Th2 balance including inhibition of lipoxygenase enzyme and other inflammatory mediators. ${ }^{24}$

The recent studies demonstrate that quercetin is more effective than cromolyn in blocking the human mast cell cytokines. $^{32}$ Oral intake of $360 \mathrm{mg}$ tomato extract that contains flavonoids for 8 weeks have been shown to reduce sneezing, rhinorrhea and nasal obstruction in AR patients. ${ }^{33}$ In one clinical trial, 20 patients with Japanese cedar pollinosis (allergic to Japanese cedar) who consumed 2 capsules of $100 \mathrm{mg}$ of enzymatically-modified isoquercitrin (EMIQ) per day for 8 weeks showed significantly lower total ocular score and ocular itching score than the placebo group $(p<0.05)$ during the pollen season. ${ }^{34}$ However, the TNSS, nasal congestion scores, lacrimation scores, ocular congestion score sand activities of daily living scores were not statistically significant. Interestingly, the results from that study were similar to the results of this preliminary data. Correspondingly, the post treatment TOSS and severity of itchy eyes were reduced remarkably in the patients received oral shallot supplement for 4 weeks. The preliminary result from this study has confirmed the efficacy and safety of oral shallot supplement as combination therapy with cetirizine in AR patients. Over $60 \%$ of patients who received oral shallot supplement combined with cetirizine demonstrated the favorable VAS more than cetirizine alone. Among allergic symptoms, itchy nose and itchy eyes seemed to be most improved when shallot capsules were added. Severity of rhinorrhea and sneezing were also reduced in the AR patients who ingested oral shallot supplement but insignificantly differed from the patients who received cetirizine alone. Thus, it is reasonable to use shallot or onion in CAM to reduce the allergic symptoms in AR patients. Further clinical trial with larger sample size should be performed to verify the efficacy and safety.

Interestingly, nasal obstruction and NAR were the only symptom and sign that could not be enhanced significantly by the cetirizine with or without shallot. These findings might correlate with the pathophysiology of nasal congestion in AR. The histaminergic pathway plays less important role than the mediators, such as leukotrienes, thromboxanes, and prostaglandin D2 (PGD2) for nasal mucosal swelling and venous engorgement. ${ }^{35}$ However, since the patients with severe nasal congestion scores were excluded from the study due to ethical issue, the effectiveness of cetirizine with or without shallot in AR remained inconclusive.

Nevertheless, the exact mechanism of how shallots help easing the allergic symptoms is not fully understood. The effects of quercetin as mast cell stabilizer prevents mast cells from degranulate the histamine. After level of histamine is decreased, nasal mucosal inflammation was reduced, thereby improvement of AR symptoms. Despite the safety profile, mast cell stabilizers such as cromones are not commonly used in the market nowadays because of their weaker effect compared to antihistamines, leukotriene receptor antagonists, and topical corticosteroids. ${ }^{26}$ Unfortunately, chromones are not available in topical nasal form in Thailand. Only the topical eye drop of nedocromil and lodoxamide are available.

One of various confounding factors that could affect the participants' AR symptoms during the post-treatment evaluation was the unavoidable allergen exposure. Patients who participated in the study during February to April were highly affected by smog, air pollution known as burning season in the Northern part of Thailand. The exposures to air pollution of final particle (PM 2.5) in the air led to direct irritation of nasal epithelium and worsen nasal and ocular symptoms in AR patients. In the same way, some patients who were allergic to house dust mites could not avoid the allergen exposure in their houses or workplaces. Large amounts of shallot capsules per day could also lead to patients' poor compliance.

Since the scientific report of shallot consumption for AR had never been made, timing of action and time to response are unknown. The dosage of daily shallot intake was derived from the wisdom knowledge of Thai folk medicine which suggests that eating of at-least 1 bulb of shallot per day is effective for AR. The consumption should not exceed 3 bulbs per day due to possible adverse effects such as memory loss or decrease strength of hair roots. ${ }^{8}$ Three grams of shallot capsules in this study was equivalent to one and a half bulb of fresh shallot. Nevertheless, this preliminary study showed that these side effects did not differ from the placebo when one and half bulbs of shallots were eaten each day for 4 weeks. Furthermore, the CNS adverse events appeared in the participants tended to be caused by cetirizine rather than oral shallot supplement. Shallots were well-tolerated by most patients.

In conclusions, Thai shallot (A.ascalonicum L.) owned similar antiallergic activity to Japanese onion (A.cepa). High contents of quercetin 4'-glucoside and quercetin 3,4'-diglucoside found in both shallot and onion might be responsible for the inhibition of type I allergy. Thus, it is reasonable to use shallot or onion in CAM to reduce allergic symptoms in allergic patients. The use of oral shallot supplement of 1 and a half bulbs per day in combination with standard dose of cetirizine in AR patients was shown to be safe and improve patients' overall symptoms more than placebo, especially for itchy nose and eyes. However, due to limitation of the sample size in this preliminary results, further clinical trial will be carried on with larger subject numbers to verify the efficacy and safety.

\section{Acknowledgements}

The in-vitro part of this study was the work under the ASEAN+3 Cross Border Research (2017) Program, granted by the International College of Digital Innovation, Chiang Mai University, Thailand. The clinical phase was supported by the Faculty of Medicine Research Fund, Chiang Mai University, Thailand. 


\section{References}

1. Pawankar R, Bunnag C, Chen Y, Fukuda T, Kim YY, Le LT, et al. Allergic rhinitis and its impact on asthma update (ARIA 2008)--western and Asian-Pacific perspective. Asian Pac J Allergy Immunol. 2009; 27(4):237-43.

2. Greiner AN, Hellings PW, Rotiroti G, Scadding GK. Allergic rhinitis. Lancet. 2011;378(9809):2112-22.

3. Naclerio RM, Bachert C, Baraniuk J. Pathophysiology of nasal congestion. Int J Gen Med. 2010;3:47-57.

4. Hon KL, Fung CK, Leung AK, Lam HS, Lee SL. Recent Patents of Complementary and Alternative Medicine for Allergic Rhinitis. Recent Pat Inflamm Allergy Drug Discov. 2015;9(2):107-19.

5. Sayin I, Cingi C, Oghan F, Baykal B, Ulusoy S. Complementary therapies in allergic rhinitis. ISRN Allergy. 2013;2013:938751.

6. Ulbricht C. Allergic Rhinitis: An Integrative Approach. Alternative and complementary therapies. 2010;16:107-11.

7. Guo R, Pittler MH, Ernst E. Herbal medicines for treatment of allergic rhinitis: a systematic review. Ann Allergy Astma Immunol. 2007;99: 483-95.

8. Pitiporn S. [Herbs for living without diseases "Thai way of heathy life with Aphaibhubet”...for self-reliance]. 16th ed. Bangkok: Poramudh publication; 2012. Thai.

9. Upadhyay RK. Nutritional and therapeutic potential of Allium vegetables. J Nutr Ther. 2017;6:18-37.

10. Mikaili P, Maadirad S, Moloudizargari M, Aghajanshakeri S, Sarahroodi S. Therapeutic uses and pharmacological properties of garlic, shallot, and their biologically active compounds". Iran J Basic Med Sci. 2013;16(10):1031-48.

11. Dhulappa M. Allium ascalonicum Linn.-An Ayuravedic perspective. Int Ayuravedic Med. 2014;2:478-82.

12. Werawattanachai N, Kaewamatawong R, Junlatat J, Sripanidkulchai B. Anti-inflammatory potential of ethanolic bulb extract of Allium ascalonicum. Sci Technol Ubon Ratchathani University. 2015;2:63-8.

13. Mohammadi-Motlagh HR, Mostafaie A, Mansouri K. Anticancer and anti-inflammatory activities of shallot (Allium ascalonicum) extract. Arch Med Sci. 2011;7:38-44.

14. Mahmoudabadi AZ, Gharib Naseri MK. Antifungal activity of shallot, Allium ascalonicum Linn. (Liliaceae), in vitro. Med Plants Res. 2009;3:450-3.

15. Wongmekiat $\mathrm{O}$, Leelarugrayub $\mathrm{N}$, Thamprasert $\mathrm{K}$. Beneficial effect of shallot (Allium ascalonicum L.) extract on cyclosporine nephrotoxicity in rats. Food Chem Toxicol. 2008;46:1844-50.

16. Swamy KRM, Gowda RV. Leek and Shallot. In: Dalam Peter KV, editor. Handbook of Herbs and Spices Volume 3. Woodhead Publishing Limited and CRC Press. Cambridge-New York; 2006. P. 381-3822006.

17. Fattorusso E, Iorizzi M, Lanzotti V, Taglialatela-Scafati O. Chemical composition of shallot (Allium ascalonicum Hort.). J Agric Food Chem. 2002;50:5686-90.

18. Ismail A, Marjan Z, Foong C. Total antioxidant activity and phenolic content in selected vegetables. Food Chemistry. 2004;87(4):581-6.

19. Kiviranta J, Huovinen K, Hiltunen R. Variation of phenolic sub-stances in onion. Acta Pharmaceut Fennica. 1988;97:67-72.
20. Terrance L, Charles G, Larry F, William KH, Jose C, Vicente N Molecular characterization of quercetin and quercetin glycosides in Allium vegetables. Their effects on malignant cell transformation. ACS Symp Ser. 1992;50:220-38.

21. Wiczkowski W, Romaszko J, Bucinski A, Szawara-Nowak D, Honke J, Zielinski $\mathrm{H}$, et al. Quercetin from shallots (Allium cepa L. var. aggregatum) is more bioavailable than its glucosides. J Nutr. 2008;138(5): $885-8$.

22. Bonaccorsi P, Caristi C, Gargiulli C, Leuzzi U. Flavonol glucosides in Allium species: A comparative study by means of HPLC-DAD-ESI-MS-MS. Food Chemistry. 2008;107(4):1668-73.

23. Sato A, Zhang T, Yonekura L, Tamura H. Antiallergic activities of eleven onions (Allium cepa) were attributed to quercetin 4'-glucoside using QuEChERS method and Pearson's correlation coefficient. J Funct Foods. 2015;14:581-9.

24. Mlcek J, Jurikova T, Skrovankova S, Sochor J. Quercetin and Its Anti-Allergic Immune Response. Molecules. J Nutr Ther. 2016;21(5):623.

25. Asada $\mathrm{T}$, Tamura $\mathrm{H}$. Isolation of bilberry anthocyanidin 3-glycosides bearing ortho-dihydroxyl groups on the $\mathrm{B}$ ring by forming an aluminum complex and their antioxidant activity. J Agric Food Chem. 2012;60(42):10634-40

26. Greiner AN, Hellings PW, Rotiroti G, Scadding GK. Allergic rhinitis Lancet. 2011;378:2112-22.

27. Guo R, Pittler MH, Ernst E. Herbal medicines for the treatment of allergic rhinitis: a systematic review. Ann Allergy Asthma Immunol. 2007;99:483-95.

28. Upadhyay RK. Nutritional and therapeutic potential of Allium vegetables. J Nutr Ther. 2017;6:18-37.

29. Bischoff SC. Quercetin: potentials in the prevention and therapy of disease. Curr Opin Clin Nutr Metab Care. 2008;11(6):733-40.

30. Kelly GS. Quercetin. Monograph. Altern Med Rev. 2011;16(2):172-94.

31. Kimata M, Shichijo M, Miura T, Serizawa I, Inagaki $N$, Nagai $H$ Effects of luteolin, quercetin and baicalein on immunoglobulin E-mediated mediator release from human cultured mast cells. Clin Exp Allergy. 2000;30(4):501-8.

32. Weng Z, Zhang B, Asadi S, Sismanopoulos N, Butcher A, Fu X, et al. Quercetin is more effective than cromolyn in blocking human mast cell cytokine release and inhibits contact dermatitis and photosensitivity in humans. PLoS One. 2012;7(3):e33805.

33. Yoshimura M, Enomoto T, Dake Y, Okuno Y, Ikeda H, Cheng L, et al. An evaluation of the clinical efficacy of tomato extract for perennial allergic rhinitis. Allergol Int. 2007;56(3):225-30.

34. Kawai M, Hirano T, Arimitsu J, Higa S, Kuwahara Y, Hagihara K. Effect of enzymatically modified isoquercitrin, a flavonoid, on symptoms of Japanese cedar pollinosis: a randomized double-blind placebo-controlled trial. Int Arch Allergy Immunol. 2009;149(4):359-68.

35. Naclerio RM, Bachert C, Baraniuk J. Pathophysiology of nasal congestion. Int J Gen Med. 2010;3:47-57. 